Estimating risk factors for three negative outcomes in pregnant women admitted to the Centre de Références et Urgences Obstétricales (CRUO) and their neonates in Port-au-Prince, Haiti between January 2013 and June 2018

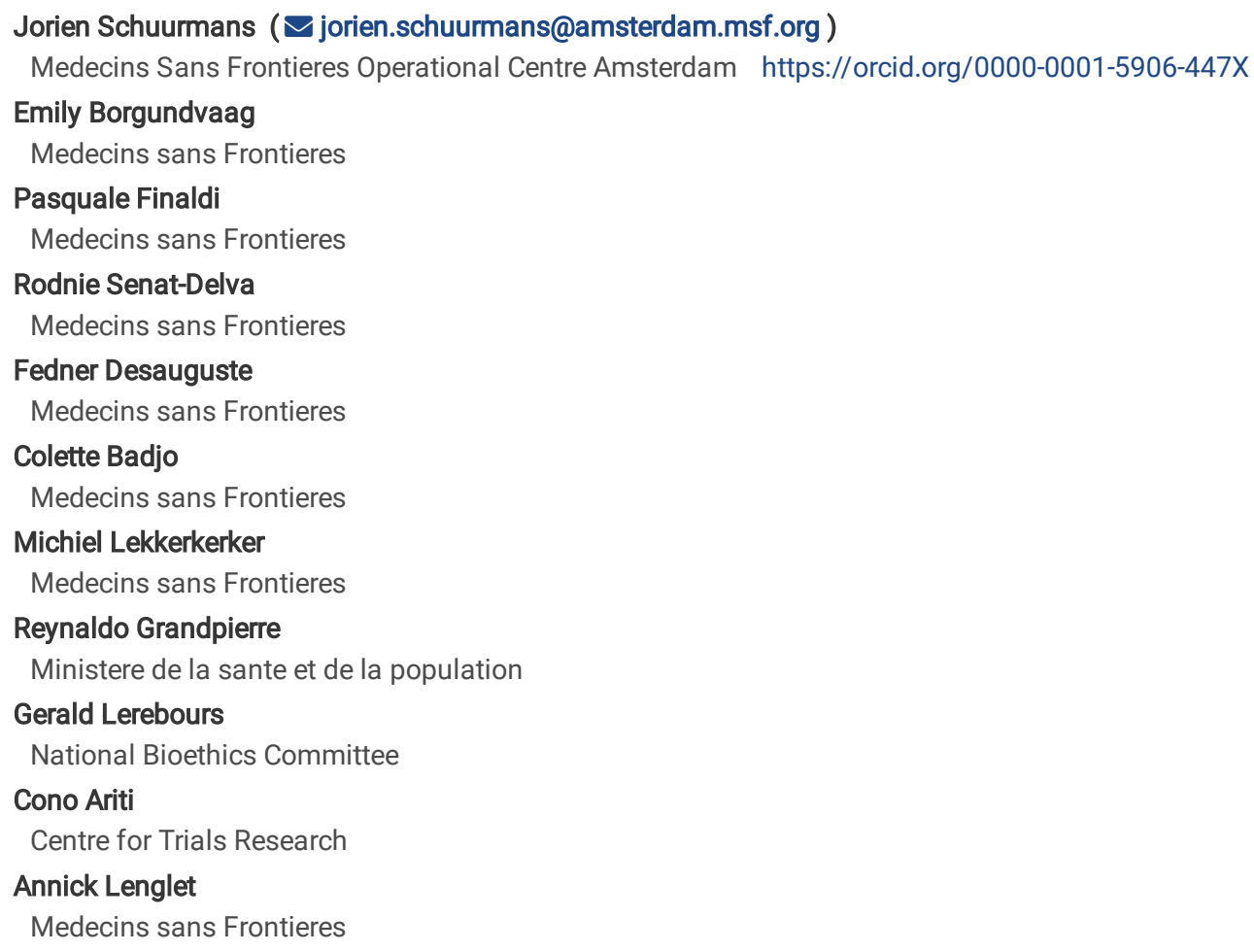




\section{Abstract Background}

The prevalence of (pre-)eclampsia in pregnant women in Haiti is high and access to maternal health services is scarce. Limited evidence exists around negative maternal and neonatal outcomes in Haitian women and their offspring. We describe the patient profile of women admitted to an obstetric emergency hospital in Port-au-Prince between January 2013 and June 2018 and the estimated risk factors for maternal death, stillbirth and low birthweight (LBW).

\section{Methods}

We calculated frequencies of age groups, singleton vs. multiple pregnancies, delivery procedures and antenatal care (ANC) services for all maternal admissions. We estimated the associated risk between these factors and the three negative outcomes by calculating odds ratios (OR) and their $95 \%$ confidence intervals $(\mathrm{Cl})$ using univariate and multivariate logistic regression. We adjusted for an interaction between delivery procedure and pregnancy category in the regression models for maternal death and stillbirth.

\section{Results}

We included 31,509 women and 24,983 deliveries and documented 204 (0.6\%) maternal deaths (648/100,000 women giving birth), 1,962 (7.9\%) stillbirths and 11,008 (44.1\%) LBW neonates. $34.9 \%$ of all admissions $(n=10,991)$ were women with (pre-)eclampsia. Maternal death was more likely in women with complicated pregnancies and/or deliveries and women with (pre-)eclampsia when undergoing a C-section compared to women with uncomplicated vaginal deliveries (OR 4.8; $\mathrm{Cl} 1.7-13.8$ and $\mathrm{OR} 2.3 ; \mathrm{Cl} 1.5-3.6$ respectively). Stillbirth was more likely in women $\geq 35$ years compared to women 20-34 years in complicated pregnancies and/or deliveries (OR 1.3; $\mathrm{Cl} 1.1-1.6)$ and (pre-)eclampsia (OR 1.4; Cl 1.2-1.7). C-sections in women with a complicated pregnancy and/or delivery and women with (pre-)eclampsia reduced the risk of stillbirth (OR 0.7; $\mathrm{Cl} 0.6-0.9$ and OR 0.3 ; $\mathrm{Cl} 0.2-0.3$ respectively). Not attending ANC was associated with a higher risk of stillbirth (OR 4.8; Cl 3.6-6.6) and LBW (OR 1.4; Cl 1.1-1.9) for women with complicated pregnancies and/or deliveries.

\section{Conclusion}

Maternal mortality in high-risk pregnancies in CRUO is higher than the national estimate of 529 per 100,000 deliveries. Attendance of ANC services is associated with a decrease in adverse neonatal outcomes including LBW and stillbirth. We recommend that access to maternal and neonatal healthcare facilities in Port-au-Prince is improved.

\section{Background}

Globally, an estimated 340,000 maternal deaths, 2.7 million stillbirths and 3.1 million neonatal deaths occur each year [1]. Low-income countries bear the greatest burden of these as they account for $98 \%$ of all maternal and child deaths [2]. Haiti is the poorest country in the Caribbean, is highly dependent on foreign aid and suffers from the highest maternal and neonatal mortality in the Western hemisphere [3,4]. While maternal and neonatal mortality rates in Haiti have reduced dramatically since the 1960s, little progress has been made in the last two decades [5, 6]. In 2018, maternal mortality rates in Haiti increased to 529 women per 100,000 giving birth and notably exceeded the number of Haiti's neighbouring country The Dominican Republic, which recorded a five-fold lower maternal mortality than Haiti [6].

The main barriers for improved maternal and neonatal health in Haiti are poverty, lack of access to health care, poor infrastructure and lack of skilled birth attendance. More than $50 \%$ of the Haitian population lives below the poverty line of less than US\$2.41 per day [7]. In addition, Haiti is suffering under the weight of a free-falling currency, a high cost of living and a 13\% annual inflation rate in 2017 [7]. It is estimated that almost $50 \%$ of the Haitian population lack access to health care because of financial and geographical barriers [4]. Infrastructure is poor and the effects of urban congestion contribute to the inability of seeking health care [8]. Other limitations to improve maternal and neonatal health and reduce mortality are inadequate health care facilities and the shortage of health workers $[4,9,10]$. In only $16 \%$ of births in Haiti a skilled birth attendant was present during delivery [8].

Factors that are associated with maternal death are multiple gestation [11], mode of delivery [12] and attending antenatal care [13]. Improving access to antenatal care decreases maternal mortality as well as negative neonatal outcomes [13]. In most low-resource countries, delivery complications that are directly associated with the high prevalence of maternal death are haemorrhage (23\%) and pre-hypertension $(22 \%)[4,10,14]$. However, in Haiti, the primary cause of maternal death is hypertensive disorders including eclampsia and pre-eclampsia (37.5\%) and postpartum haemorrhage (22\%) [4, 10, 14]. A recent study conducted in a hospital in rural Haiti found a prevalence of (pre-)eclampsia of 16.7\% in women seeking care [14]. Haitian women have been shown to have higher blood pressures compared to women in other Caribbean countries, which could be a risk factor for this high prevalence of (pre-)eclampsia [4]. In Haiti, the association between poor antenatal care access and maternal mortality is unclear, but it is known that the antenatal care uptake in Haiti remains low with $67 \%$ accessing the recommended four ANC visits [15]. 
Stillbirths are frequently associated with an early gestational age, placental abruption, maternal death and complications from prolonged labour. Other risk factors for stillbirth are women not attending ANC [16], multiple pregnancy [17] and advanced maternal age [18]. Multiple studies show that the number of stillbirths could be reduced by improved (access to) obstetric care during labor and delivery and C-sections are often needed to reduce this risk $[15,16]$. There are no reliable estimates on the incidence of stillbirth in Haiti.

The pre-term birth rate in Haiti was $14.1 \%$ in 2010 and the average percentage of neonates with low birthweight (LBW) was $23 \%$ [4]. Neonates with a LBW are 20 times more likely to die than neonates with a normal birthweight [4]. Research has shown that mothers under the age of 20 have higher risks of prematurity and LBW $[19,20]$. Furthermore, plurality of birth is associated with LBW. However, these findings were mainly attributed to the effects of a lower gestational age in twins and triplets than in singletons [21]. Neonates with LBW are more likely to be delivered by a C-section as this delivery type reduces the risk of neonatal mortality [22].

Between 2011 and 2018, Médécins sans Frontières operated an obstetric emergency hospital and neonatal care unit in Port-au-Prince (the capital of Haiti) targeted at women with high risk pregnancies. Even though maternal health is a public health priority in Haiti, very little is known about the major factors that lead to adverse pregnancy outcomes in women with a complicated pregnancy or delivery and with (pre-) eclampsia in Haiti. For this reason, we analysed maternal admission data collected between 2013 and 2018 to examine risk factors for maternal death, stillbirth and low birthweight neonates in this cohort. The results from this study will present the opportunity to advise healthcare policies to mitigate risk factors affecting pregnancy outcomes amongst Haitian women.

\section{Methods}

\section{Setting and design of the study}

The study was conducted in the "Centre de Référence pour les Urgences Obstétricales" (CRUO) in Haiti, which was established by Médecins Sans Frontières (MSF) in March 2011 in response to the alarming maternal mortality indicators and pervasive violence in Port-au-Prince, Haiti. Since then, MSF provided free emergency care to women with high-risk pregnancies and care for the neonates born to these women. CRUO was the main facility for obstetric and neonatal care in metropolitan Port-au-Prince. The hospital closed in July 2018. We conducted a retrospective cohort study for all maternal admissions in CRUO between January 2013 and June 2018 to estimate the most common risk factors for maternal death, stillbirth and LBW neonates.

\section{Study participants}

We included all pregnant women who were admitted to CRUO and their neonates in the study period. We excluded all maternal admissions from suspected cholera.

\section{Procedures and definitions}

We defined a maternal death as any pregnancy-related admission resulting in direct maternal death. Stillbirth was defined as any infant born at gestational age of 22 weeks or more or weighing more than 500 grams not showing signs of life at time of delivery. A LBW neonate was any infant with a birthweight below 2,500 grams [23].

We explored the following risk factors for negative outcomes in the analysis: age group for admitted women $(<20$ years; between $20-34$ years; $\geq 35$ years), a singleton or multiple neonate (twins or triplets), delivery procedure (uncomplicated vaginal delivery, complicated non-instrumental vaginal delivery instrumental vaginal delivery or C-section) and attending ANC. An uncomplicated vaginal delivery was defined as a term pregnancy, cephalic, singleton, vaginal delivery (including cases with minimal or no assistance, with or without episiotomy). A complicated non-instrumental vaginal delivery was defined as all non-instrumental vaginal deliveries that do not fit the definition of normal spontaneous vaginal delivery (e.g., augmentation of labor, breech delivery, multiple pregnancy, and any instrumental manipulation or assistance). An instrumental vaginal delivery was defined as any delivery assisted by use of forceps or vacuum extraction. A C-section was defined as any delivery conducted by caesarian section.

We assessed the three negative outcomes in three maternal groups: uncomplicated pregnancy and/or delivery, (pre)-eclamptic women and complicated pregnancy and/or delivery. Uncomplicated pregnancies and/or deliveries were defined as a singleton pregnancy without maternal morbidities, LBW or delivery complications. An eclamptic maternity admission was defined as a pregnant woman admitted to CRUO who experienced convulsive seizures occurring in the context of pre-eclampsia without other cause between 20 weeks of pregnancy and early postpartum. Pre-eclampsia was defined as hypertension (with diastolic blood pressures constantly greater than $90 \mathrm{mmHg}$ ), proteinuria (greater than ++ of protein on urine dipstick) and upper body edema that appears suddenly or rapidly worsens. A complicated pregnancy and/or delivery was defined as all complicated pregnancies with exception of eclampsia and pre-eclampsia, with or without delivery complications. This included pregnancy complications such as ante-partum hemorrhage, premature labor, scarred uterus, ante-partum infection, hypertension, fetal distress; and/or delivery complications including post-partum hemorrhage, obstructed labor, ruptured uterus and other complications.

\section{Statistical analysis}

Maternal admissions were described by frequencies and proportions, calculated by exposure. We performed Chi square tests to test whether the frequencies of negative maternal outcomes differed between pregnancy categories. Univariable associations were calculated using logistic regression models. The analysis was stratified by pregnancy category (complicated pregnancy and/or delivery and (pre-) eclampsia), resulting in a separate logistic regression model used for each pregnancy category. Women with an uncomplicated pregnancy and/or delivery were excluded for the regression

Page $3 / 13$ 
analysis as none of the negative outcomes occurred in this group of patients. For the regression model for LBW, the delivery category was excluded due to possible reverse causality.

To identify the effect of multiple risk factors, multivariate logistic regression for each strata of pregnancy category was conducted using backward elimination, meaning that all independent variables were entered into the model and variables were deleted one at a time if they were not significantly associated with the outcome $(\mathrm{p}<0.05)$. In order to investigate the interaction between delivery procedure and pregnancy category, and associations with the other potential risk factors, an interaction term between delivery procedure and pregnancy category was introduced into the regression models. This was done for all outcomes except for LBW.

In CRUO, maternal admission criteria were restricted to higher risk maternal groups in 2013 and 2018 compared to other years. In order to verify that this admission criteria restriction did not lead to over- or underestimation of risk factors in the overall analysis, we conducted a sensitivity analysis. This was done by repeating the same analysis in four different time periods: 1) including all years from 2013 to 2018; 2) excluding 2013 and 2018; 3 ) excluding 2013 and 4) excluding 2018.

All data cleaning and data analysis was performed with Stata $15{ }^{\circledR}$.

\section{Results}

A total of 31,509 women were admitted to CRUO in the period between January 2013 to June 2018 . We excluded 8 observations due to poor data quality and included 31,501 maternal admissions to describe the cohort and estimate risk factors for maternal death. In the same period, a total number of 24,978 deliveries were recorded. We included all 24,978 deliveries to estimate risk factors for stillbirth, but included 24,938 deliveries for the analysis around LBW (40 deliveries were excluded due to poor data quality on birthweight of the neonate).

\section{Descriptive analysis by outcome}

We included all years in the descriptive analysis and demonstrated the outcomes by pregnancy category in Table 1 . The average number of admissions per month was 588 (median $=578, \mathrm{SD}=94$ ). The majority of the women admitted experienced a complicated pregnancy and/or delivery $(n=15,655$; $49.7 \%$ ), while 10,990 women (34.9\%) had eclampsia or pre-eclampsia and 4,856 (15.1\%) experienced an uncomplicated pregnancy and/or delivery. Two hundred-four ( $0.6 \%$ ) maternal deaths occurred, of which $1.1 \%$ of the women with (pre-) eclampsia died and $0.5 \%$ of the women with a complicated pregnancy and/or delivery. A total of 1,962 deliveries (7.9\%) resulted in at least one stillbirth; $9.4 \%$ of all women with a complicated pregnancy and/or delivery had a stillbirth and $10.0 \%$ of all women with (pre-) eclampsia had a stillbirth. For LBW, 11,008 mothers (44.1\%) delivered at least one neonate with LBW. The proportion of neonates with LBW was $58.5 \%$ in women with (pre-) eclampsia and $50.8 \%$ in women with a complicated pregnancy and/or delivery. None of the uncomplicated pregnancies and/or deliveries were associated with maternal death, stillbirth or LBW. Women with (pre-) eclampsia were more likely to die and to have a neonate with LBW than women with a complicated pregnancy and/or delivery. There was no significant difference in the proportion of stillbirth between women with a complicated pregnancy and/or delivery and women with (pre-) eclampsia.

Table 1

Descriptive analysis of outcomes stratified by pregnancy category

\begin{tabular}{|c|c|c|c|c|c|c|c|c|c|c|}
\hline & & \multicolumn{2}{|c|}{ All pregnancies } & \multicolumn{2}{|c|}{$\begin{array}{l}\text { Uncomplicated } \\
\text { pregnancy/delivery }\end{array}$} & \multicolumn{2}{|c|}{$\begin{array}{l}\text { Complicated } \\
\text { pregnancy/delivery }\end{array}$} & \multicolumn{2}{|c|}{ (Pre) Eclampsia } & \multirow[t]{2}{*}{$\begin{array}{l}\text { p- } \\
\text { value }\end{array}$} \\
\hline & & $\mathrm{n}$ & $\%$ & $\mathrm{n}$ & $\%$ & $\mathrm{n}$ & $\%$ & $\mathrm{n}$ & $\%$ & \\
\hline \multirow{3}{*}{$\begin{array}{l}\text { Maternal } \\
\text { death }\end{array}$} & Yes & 204 & $0.6 \%$ & 0 & $0.0 \%$ & 85 & $0.5 \%$ & 119 & $1.1 \%$ & \multirow{3}{*}{$<.001$} \\
\hline & No & 31,297 & $99.4 \%$ & 4,856 & $100.0 \%$ & 15,570 & $99.5 \%$ & 10,871 & $98.9 \%$ & \\
\hline & Total & 31,501 & $100.0 \%$ & 4,856 & $100.0 \%$ & 15,655 & $100.0 \%$ & 10,990 & $100.0 \%$ & \\
\hline \multirow[t]{3}{*}{ Stillbirth } & Yes & 1,962 & $7.9 \%$ & 0 & $0.0 \%$ & 929 & $9.4 \%$ & 1,033 & $10.0 \%$ & \multirow[t]{3}{*}{0.16} \\
\hline & No & 23,016 & $92.1 \%$ & 4,842 & $100.0 \%$ & 8,912 & $90.6 \%$ & 9,262 & $90.0 \%$ & \\
\hline & Total & 24,978 & $100.0 \%$ & 4,842 & $100.0 \%$ & 9,841 & $100.0 \%$ & 10,295 & $100.0 \%$ & \\
\hline \multirow[t]{3}{*}{ LBW } & Yes & 11,008 & $44.1 \%$ & 0 & $0.0 \%$ & 4,992 & $50.8 \%$ & 6,016 & $58.5 \%$ & \multirow{3}{*}{$<.001$} \\
\hline & No & 13,930 & $55.9 \%$ & 4,838 & $100.0 \%$ & 4,833 & $49.2 \%$ & 4,259 & $41.5 \%$ & \\
\hline & Total & 24,938 & $100.0 \%$ & 4,838 & $100.0 \%$ & 9,825 & $100.0 \%$ & 10,275 & $100.0 \%$ & \\
\hline
\end{tabular}

\section{Regression analysis}

\section{Maternal death}


Table 2 displays the results of the univariate and multivariate logistic regression models for maternal death. C-section was a risk factor for maternal death in women with a complicated pregnancy and/or delivery as well as in women with (pre-) eclampsia. After including an interaction term for delivery procedure and pregnancy category, the model showed that women with a complicated pregnancy and/or delivery had 4.81 times more likely of dying when having a C-section compared to a normal delivery (OR 4.81; Cl 1.67-13.84). Women with (pre-) eclampsia were 2.3 times more likely of dying when having a $\mathrm{C}$-section compared to a normal delivery (OR 2.30; $\mathrm{Cl} 1.46-3.61$ ). Women with (pre-) eclampsia were 5.62 times more likely to die than women with a complicated pregnancy and/or delivery. 
Table 2

Univariate and multivariate regression analysis maternal death

\begin{tabular}{|c|c|c|c|c|c|c|c|c|c|c|}
\hline \multirow{3}{*}{ Risk factor } & \multicolumn{4}{|c|}{ Complicated pregnancy/delivery } & \multicolumn{4}{|c|}{ (Pre) eclampsia } & \multirow{2}{*}{\multicolumn{2}{|c|}{$\begin{array}{l}\text { All pregnancies } \\
\begin{array}{l}\text { Multivariate } \\
\text { analysis }\end{array}\end{array}$}} \\
\hline & \multirow{2}{*}{$\begin{array}{l}\text { Deaths } \\
85 \\
(0.6 \%)\end{array}$} & \multicolumn{2}{|l|}{$\begin{array}{l}\text { Univariate } \\
\text { analysis }\end{array}$} & $\begin{array}{l}\text { Multivariate } \\
\text { analysis }\end{array}$ & \multirow{2}{*}{$\begin{array}{l}\text { Deaths } \\
119 \\
(1.1 \%)\end{array}$} & \multicolumn{2}{|l|}{$\begin{array}{l}\text { Univariate } \\
\text { analysis }\end{array}$} & $\begin{array}{l}\text { Multivariate } \\
\text { analysis }\end{array}$ & & \\
\hline & & $\begin{array}{l}\text { OR } \\
(95 \% \mathrm{Cl})\end{array}$ & $p$ & $\begin{array}{l}\text { OR } \\
(95 \% \mathrm{Cl})\end{array}$ & & $\begin{array}{l}\text { OR } \\
(95 \% \mathrm{Cl})\end{array}$ & $\mathbf{p}$ & $\begin{array}{l}\text { OR } \\
(95 \% \mathrm{Cl})\end{array}$ & $\begin{array}{l}\text { OR } \\
(95 \% \mathrm{Cl})\end{array}$ & $\mathrm{p}$ \\
\hline Age Group & & & 0.14 & & & & 0.24 & & & \\
\hline$<20$ & $\begin{array}{l}4 \\
(0.3 \%)\end{array}$ & $\begin{array}{l}0.52 \\
(0.19- \\
1.43)\end{array}$ & & * & $\begin{array}{l}11 \\
(1.0 \%)\end{array}$ & $\begin{array}{l}0.87 \\
(0.46- \\
1.63)\end{array}$ & 0.65 & * & * & \\
\hline $20-34$ & $\begin{array}{l}58 \\
(0.5 \%)\end{array}$ & reference & & reference & $\begin{array}{l}87 \\
(1.2 \%)\end{array}$ & reference & & reference & reference & \\
\hline$\geq 35$ & $\begin{array}{l}23 \\
(0.7 \%)\end{array}$ & $\begin{array}{l}1.34 \\
(0.83- \\
2.18)\end{array}$ & & * & $\begin{array}{l}21 \\
(0.8 \%)\end{array}$ & $\begin{array}{l}0.67 \\
(0.42- \\
1.09)\end{array}$ & 0.11 & * & * & \\
\hline Multiple pregnancy & & & 0.59 & & & & 0.047 & & & \\
\hline Singleton & $\begin{array}{l}28 \\
(0.3 \%)\end{array}$ & reference & & reference & $\begin{array}{l}81 \\
(0.8 \%)\end{array}$ & reference & & reference & reference & \\
\hline Multiple & $\begin{array}{l}2 \\
(0.4 \%)\end{array}$ & $\begin{array}{l}1.49 \\
(0.35- \\
6.26)\end{array}$ & & * & $\begin{array}{l}7 \\
(1.8 \%)\end{array}$ & $\begin{array}{l}2.20 \\
(1.01- \\
4.79)\end{array}$ & & * & * & \\
\hline Delivery procedure & & & 0.003 & 0.003 & & & $<.001$ & $<.001$ & & $<.001$ \\
\hline Normal vaginal & $\begin{array}{l}4 \\
(0.1 \%)\end{array}$ & reference & & reference & $\begin{array}{l}28 \\
(0.5 \%)\end{array}$ & reference & & reference & reference & \\
\hline $\begin{array}{l}\text { Complicated non- } \\
\text { instrumental }\end{array}$ & $\begin{array}{l}1 \\
(0.3 \%)\end{array}$ & $\begin{array}{l}2.73 \\
(0.30- \\
24.46)\end{array}$ & & $\begin{array}{l}2.73 \\
(0.30- \\
24.46)\end{array}$ & $\begin{array}{l}0 \\
(0.0 \%)\end{array}$ & $\begin{array}{l}\text { *No matern } \\
\text { deaths }\end{array}$ & nal & $\begin{array}{l}\text { *No maternal } \\
\text { deaths }\end{array}$ & $0.51(0.07$ & $7-3.74)$ \\
\hline Instrumental vaginal & $\begin{array}{l}0 \\
(0.0 \%)\end{array}$ & $\begin{array}{l}\text { *No materna } \\
\text { deaths }\end{array}$ & & $\begin{array}{l}\text { *No maternal } \\
\text { deaths }\end{array}$ & $\begin{array}{l}0 \\
(0.0 \%)\end{array}$ & $\begin{array}{l}\text { *No matern } \\
\text { deaths }\end{array}$ & nal & $\begin{array}{l}\text { *No maternal } \\
\text { deaths }\end{array}$ & * & \\
\hline Caesarean section & $\begin{array}{l}25 \\
(0.5 \%)\end{array}$ & $\begin{array}{l}4.78 \\
(1.66- \\
13.76)\end{array}$ & & $\begin{array}{l}4.78 \\
(1.66- \\
13.76)\end{array}$ & $\begin{array}{l}60 \\
(1.2 \%)\end{array}$ & $\begin{array}{l}2.36 \\
(1.51- \\
3.71)\end{array}$ & & $\begin{array}{l}2.36 \\
(1.51- \\
3.71)\end{array}$ & $\begin{array}{l}2.45 \\
(1.63- \\
3.69)\end{array}$ & \\
\hline Ante-natal care & & & 0.42 & & & & 0.11 & & & 0.64 \\
\hline Yes & $\begin{array}{l}78 \\
(0.6 \%)\end{array}$ & reference & & reference & $\begin{array}{l}113 \\
(1.1 \%)\end{array}$ & reference & & reference & reference & \\
\hline No & $\begin{array}{l}7 \\
(0.4 \%)\end{array}$ & $\begin{array}{l}0.73 \\
(0.33- \\
1.58)\end{array}$ & & * & $\begin{array}{l}6 \\
(2.1 \%)\end{array}$ & $\begin{array}{l}1.97 \\
(0.86- \\
4.50)\end{array}$ & & * & $\begin{array}{l}0.71 \\
(0.18- \\
2.90)\end{array}$ & \\
\hline Pregnancy type & & & & & & & & & & 0.001 \\
\hline $\begin{array}{l}\text { Complicated } \\
\text { pregnancy }\end{array}$ & & & & & & & & & reference & \\
\hline $\begin{array}{l}\text { Eclampsia/pre- } \\
\text { eclampsia }\end{array}$ & & & & & & & & & $\begin{array}{l}5.62 \\
(1.97- \\
16.06)\end{array}$ & \\
\hline \multicolumn{11}{|c|}{ Delivery procedure \# pregnancy type } \\
\hline $\begin{array}{l}\text { C-section vs normal } \\
\text { delivery \# complicated } \\
\text { pregnancy }\end{array}$ & & & & & & & & & $\begin{array}{l}4.81 \\
(1.67- \\
13.84)\end{array}$ & 0.004 \\
\hline $\begin{array}{l}\text { C-section vs normal } \\
\text { delivery \# eclampsia }\end{array}$ & & & & & & & & & $\begin{array}{l}2.30 \\
(1.46- \\
3.61)\end{array}$ & $\dot{0.001}$ \\
\hline
\end{tabular}

Table 2 Univariate and multivariate regression analysis maternal death

Stillbirth 
Multivariate analysis shows that women over the age of 35 were more likely of having a stillbirth in complicated pregnancies and/or deliveries (OR 1.31; $\mathrm{Cl} 1.12-1.55)$ and (pre-) eclampsia (OR 1.43; Cl 1.24-1.65) compared to women between 20 and 34 years old. Women under the age of 20 were less likely of having a stillbirth when they had (pre-) eclampsia (OR $0.74 ; \mathrm{Cl} 0.57-0.97$ ) compared to women between 20 and 34 years old.

No significant association between stillbirth and multiple pregnancy has been demonstrated in any group. Not attending ANC services was a risk factor for stillbirth in women with complicated pregnancies (OR 4.82; $\mathrm{Cl} 3.55-6.55)$. Table 3 displays the results of the univariate and multivariate regression analysis for stillbirth stratified by pregnancy category as well as an interaction term for delivery procedure and pregnancy category. The complete multivariate model shows that women with (pre-) eclampsia had a 1.34 times higher risk of having a stillbirth compared to women that had a complicated pregnancy and/or delivery (OR 1.34; Cl 1.18-1.52). 
Table 3

Univariate and multivariate regression analysis stillbirth

\begin{tabular}{|c|c|c|c|c|c|c|c|c|c|c|c|c|}
\hline \multirow{3}{*}{ Risk factor } & \multicolumn{5}{|c|}{ Complicated pregnancy/delivery } & \multicolumn{5}{|c|}{ (Pre-) eclampsia } & \multirow{2}{*}{\multicolumn{2}{|c|}{$\begin{array}{l}\text { All pregnancies } \\
\text { Multivariate } \\
\text { analysis }\end{array}$}} \\
\hline & \multirow{2}{*}{$\begin{array}{l}\text { Stillbirth } \\
896 \\
(9.3 \%)\end{array}$} & \multicolumn{2}{|l|}{$\begin{array}{l}\text { Univariate } \\
\text { analysis }\end{array}$} & \multicolumn{2}{|c|}{$\begin{array}{l}\text { Multivariate } \\
\text { analysis }\end{array}$} & \multirow{2}{*}{$\begin{array}{l}\text { Stillbirth } \\
\begin{array}{l}1,033 \\
(10.0 \%)\end{array}\end{array}$} & \multicolumn{2}{|l|}{$\begin{array}{l}\text { Univariate } \\
\text { analysis }\end{array}$} & \multicolumn{2}{|l|}{$\begin{array}{l}\text { Multivariate } \\
\text { analysis }\end{array}$} & & \\
\hline & & $\begin{array}{l}\text { OR } \\
(95 \% \mathrm{Cl})\end{array}$ & p & $\begin{array}{l}\text { OR } \\
(95 \% \mathrm{Cl})\end{array}$ & p & & $\begin{array}{l}\text { OR } \\
(95 \% \mathrm{Cl})\end{array}$ & p & OR $(95 \% \mathrm{Cl})$ & p & $\begin{array}{l}\text { OR } \\
(95 \% \mathrm{Cl})\end{array}$ & p \\
\hline Age Group & & & $<0.001$ & & $\begin{array}{l}<.001 \\
0.00\end{array}$ & & & $<0.001$ & & $\begin{array}{l}< \\
0.001\end{array}$ & & $\begin{array}{l}< \\
0.001\end{array}$ \\
\hline$<20$ & $\begin{array}{l}71 \\
(7.5 \%)\end{array}$ & $\begin{array}{l}0.81 \\
(0.63- \\
1.05)\end{array}$ & & $\begin{array}{l}0.78 \\
(0.60- \\
1.01)\end{array}$ & & $\begin{array}{l}67 \\
(6.7 \%)\end{array}$ & $\begin{array}{l}0.70 \\
(0.54- \\
0.91)\end{array}$ & & $\begin{array}{l}0.74(0.57- \\
0.97)\end{array}$ & & $\begin{array}{l}0.73 \\
(0.60- \\
0.88)\end{array}$ & \\
\hline $20-34$ & $\begin{array}{l}628 \\
(9.1 \%)\end{array}$ & reference & & reference & & $\begin{array}{l}640 \\
(9.3 \%)\end{array}$ & reference & & reference & & reference & \\
\hline$\geq 35$ & $\begin{array}{l}230 \\
(11.6 \%)\end{array}$ & $\begin{array}{l}1.31 \\
(1.12- \\
1.54)\end{array}$ & & $\begin{array}{l}1.31 \\
(1.12- \\
1.55)\end{array}$ & & $\begin{array}{l}326 \\
(13.4 \%)\end{array}$ & $\begin{array}{l}1.50 \\
(1.30- \\
1.73)\end{array}$ & & $\begin{array}{l}1.43(1.24- \\
1.65)\end{array}$ & & $\begin{array}{l}1.38 \\
(1.24- \\
1.54)\end{array}$ & \\
\hline $\begin{array}{l}\text { Multiple } \\
\text { pregnancy }\end{array}$ & & & 0.7 & & & & & 0.2 & & & & \\
\hline Singleton & $\begin{array}{l}884 \\
(9.4 \%)\end{array}$ & reference & & reference & & $\begin{array}{l}1,001 \\
(10.1 \%)\end{array}$ & reference & & reference & & reference & \\
\hline Multiple & $\begin{array}{l}45 \\
(10.0 \%)\end{array}$ & $\begin{array}{l}1.06 \\
(0.78- \\
1.46)\end{array}$ & & * & & $\begin{array}{l}32 \\
(8.1 \%)\end{array}$ & $\begin{array}{l}0.79 \\
(0.55- \\
1.14)\end{array}$ & & * & & * & \\
\hline $\begin{array}{l}\text { Delivery } \\
\text { procedure }\end{array}$ & & & $\begin{array}{l}< \\
0.001\end{array}$ & & $<0.001$ & & & $<0.001$ & & $\begin{array}{l}< \\
0.001\end{array}$ & & $<.001$ \\
\hline $\begin{array}{l}\text { Normal } \\
\text { vaginal }\end{array}$ & $\begin{array}{l}444 \\
(10.9 \%)\end{array}$ & reference & & reference & & $\begin{array}{l}759 \\
(14.4 \%)\end{array}$ & reference & & reference & & reference & \\
\hline $\begin{array}{l}\text { Complicated } \\
\text { non- } \\
\text { instrumental }\end{array}$ & $\begin{array}{l}49 \\
(13.1 \%)\end{array}$ & $\begin{array}{l}1.23 \\
(0.90- \\
1.69)\end{array}$ & & $\begin{array}{l}1.20 \\
(0.87- \\
1.65)\end{array}$ & & $\begin{array}{l}52 \\
(26.5 \%)\end{array}$ & $\begin{array}{l}2.14 \\
(1.55- \\
2.97)\end{array}$ & & $\begin{array}{l}2.11(1.52- \\
2.93)\end{array}$ & & $\begin{array}{l}1.43 \\
(1.15- \\
1.80)\end{array}$ & \\
\hline $\begin{array}{l}\text { Instrumental } \\
\text { vaginal }\end{array}$ & $1(3.6 \%)$ & $\begin{array}{l}0.30 \\
(0.04- \\
2.24)\end{array}$ & & $\begin{array}{l}0.30 \\
(0.04- \\
2.21)\end{array}$ & & $\begin{array}{l}4 \\
(12.1 \%)\end{array}$ & $\begin{array}{l}0.82 \\
(0.29- \\
2.33)\end{array}$ & & $\begin{array}{l}0.80(0.28- \\
2.28)\end{array}$ & & $0.60(0.24-$ & 1.50) \\
\hline $\begin{array}{l}\text { Caesarean } \\
\text { section }\end{array}$ & $\begin{array}{l}435 \\
(8.1 \%)\end{array}$ & $\begin{array}{l}0.72 \\
(0.63- \\
0.83)\end{array}$ & & $\begin{array}{l}0.71 \\
(0.62- \\
0.83)\end{array}$ & & $\begin{array}{l}218 \\
(4.5 \%)\end{array}$ & $\begin{array}{l}0.28 \\
(0.24- \\
0.33)\end{array}$ & & $\begin{array}{l}0.29(0.25- \\
0.34)\end{array}$ & & $\begin{array}{l}0.47 \\
(0.42- \\
0.51)\end{array}$ & \\
\hline Ante-natal care & & & $<.001$ & & $\begin{array}{l}<.001 \\
0.00\end{array}$ & & & 0.25 & & & & $\dot{0} .001$ \\
\hline Yes & $\begin{array}{l}865 \\
(9.05)\end{array}$ & reference & & reference & & $\begin{array}{l}1,003 \\
(10.0 \%)\end{array}$ & reference & & reference & & reference & \\
\hline No & $\begin{array}{l}64 \\
(31.8 \%)\end{array}$ & $\begin{array}{l}4.74 \\
(3.49- \\
6.43)\end{array}$ & & $\begin{array}{l}4.82 \\
(3.55- \\
6.55)\end{array}$ & & $\begin{array}{l}30 \\
(12.2 \%)\end{array}$ & $\begin{array}{l}1.25 \\
(0.85- \\
1.85)\end{array}$ & & * & & $\begin{array}{l}2.64 \\
(2.08- \\
3.34)\end{array}$ & \\
\hline $\begin{array}{l}\text { Pregnancy } \\
\text { type }\end{array}$ & & & & & & & & & & & & $\begin{array}{l}< \\
0.001\end{array}$ \\
\hline $\begin{array}{l}\text { Complicated } \\
\text { pregnancy }\end{array}$ & & & & & & & & & & & reference & \\
\hline $\begin{array}{l}\text { Eclampsia/pre- } \\
\text { eclampsia }\end{array}$ & & & & & & & & & & & $\begin{array}{l}1.34 \\
(1.18- \\
1.52)\end{array}$ & \\
\hline Delivery procedu & e \# pregna & cy type & & & & & & & & & & \\
\hline $\begin{array}{l}\text { C-section vs } \\
\text { non- } \\
\text { instrumental } \\
\text { delivery \# } \\
\text { complicated } \\
\text { pregnancy }\end{array}$ & & & & & & & & & & & $\begin{array}{l}0.59 \\
(0.43- \\
0.81)\end{array}$ & 0.001 \\
\hline
\end{tabular}




\begin{tabular}{|c|c|c|c|}
\hline \multirow[b]{2}{*}{$\begin{array}{l}\text { C-section vs } \\
\text { normal } \\
\text { delivery \# } \\
\text { complicated } \\
\text { pregnancy }\end{array}$} & \multirow[t]{2}{*}{ (Pre-) eclampsia } & \multicolumn{2}{|c|}{ All pregnancies } \\
\hline & & $\begin{array}{l}0.71 \\
(0.62- \\
0.82)\end{array}$ & $\begin{array}{l}< \\
0.001\end{array}$ \\
\hline $\begin{array}{l}\text { C-section vs } \\
\text { non- } \\
\text { instrumental } \\
\text { delivery \# } \\
\text { eclampsia }\end{array}$ & & $\begin{array}{l}0.41 \\
(0.20- \\
0.86)\end{array}$ & 0.02 \\
\hline $\begin{array}{l}\text { C-section vs } \\
\text { normal } \\
\text { delivery \# } \\
\text { eclampsia }\end{array}$ & & $\begin{array}{l}0.29 \\
(0.24- \\
0.33)\end{array}$ & $\begin{array}{l}<.001 \\
0.001\end{array}$ \\
\hline
\end{tabular}

Women that had a C-section had a lower chance of having a stillbirth in both pregnancy categories. Women with a complicated pregnancy and/or delivery who had a C-section had a 1.41 times lower chance of having a stillbirth (OR $0.71 ; \mathrm{Cl} 0.62-0.83)$ compared to a normal delivery. Women with (pre-) eclampsia had a 3.45 times lower chance (OR 0.29 ; $\mathrm{Cl} 0.25-0.34)$ of a stillbirth when they had a C-section compared to a normal vaginal delivery.

Women with a complicated pregnancy had a 1.69 higher risk of having a stillbirth when they had a complicated non-instrumental delivery compared to a C-section (OR 0.59; $\mathrm{Cl} 0.43-0.81$ ). In addition, women with (pre-) eclampsia were 2.44 times more likely to have a stillbirth when they had a complicated non-instrumental delivery compared to a C-section (OR $0.41 ; \mathrm{Cl} 0.20-0.86$ ).

Table 3 Univariate and multivariate regression analysis stillbirth

\section{LBW}

Univariate and multivariate analysis show that maternal age was significantly associated with having a neonate with LBW in women with (pre-) eclampsia. Women that experienced eclampsia or pre-eclampsia had a lower risk of having a neonate with LBW when they were under the age of 20 (OR 0.73 ; $\mathrm{Cl} 0.63-0.83$ ). Multiple pregnancy was a risk factor for LBW in women with a complicated pregnancy and/or delivery and women with (pre-) eclampsia with ORs exceeding 5. Not attending ANC was a risk factor for LBW in women with a complicated pregnancy (OR 1.40; 1.05-1.86). Table 4 displays the results of the univariate and multivariate regression analysis for LBW stratified by pregnancy category. 
Table 4

Univariate and multivariate regression analysis LBW

\begin{tabular}{|c|c|c|c|c|c|c|c|c|c|c|}
\hline \multirow{3}{*}{ Risk factor } & \multicolumn{5}{|c|}{ Complicated pregnancy/delivery } & \multicolumn{5}{|c|}{ (Pre) eclampsia } \\
\hline & \multirow{2}{*}{$\begin{array}{l}\text { LBW } \\
4,992 \\
(\mathbf{5 0 . 8 \% )}\end{array}$} & \multicolumn{2}{|c|}{ Univariate analysis } & \multicolumn{2}{|c|}{ Multivariate analysis } & \multirow{2}{*}{$\begin{array}{l}\text { LBW } \\
\mathbf{6 , 0 1 6} \\
(\mathbf{5 8 . 6 \% )}\end{array}$} & \multicolumn{2}{|c|}{ Univariate analysis } & \multicolumn{2}{|c|}{ Multivariate analysis } \\
\hline & & OR $(95 \% \mathrm{Cl})$ & $\mathbf{p}$ & OR $(95 \% \mathrm{Cl})$ & p & & OR $(95 \% \mathrm{Cl})$ & $\mathbf{p}$ & OR $(95 \% \mathrm{Cl})$ & $\mathrm{p}$ \\
\hline Age Group & & & 0.07 & & 0.07 & & & $\begin{array}{l}< \\
0.001\end{array}$ & & <. 001 \\
\hline$<20$ & $\begin{array}{l}512 \\
(54.2 \%)\end{array}$ & $\begin{array}{l}1.17(1.02- \\
1.34)\end{array}$ & & $\begin{array}{l}1.22(1.06- \\
1.40)\end{array}$ & & $\begin{array}{l}504 \\
(50.7 \%)\end{array}$ & $\begin{array}{l}0.71(0.62- \\
0.81)\end{array}$ & & $\begin{array}{l}0.73(0.63- \\
0.83)\end{array}$ & \\
\hline $20-34$ & $\begin{array}{l}3,472 \\
(50.3 \%)\end{array}$ & reference & & reference & & $\begin{array}{l}4,053 \\
(59.2 \%)\end{array}$ & reference & & reference & \\
\hline$\geq 35$ & $\begin{array}{l}1,008 \\
(50.9 \%)\end{array}$ & $\begin{array}{l}1.02(0.92- \\
1.13)\end{array}$ & & $\begin{array}{l}1.04(0.94- \\
1.15)\end{array}$ & & $\begin{array}{l}1,459 \\
(60.0 \%)\end{array}$ & $\begin{array}{l}1.04(0.94- \\
1.14)\end{array}$ & & $\begin{array}{l}1.04(0.95- \\
1.14)\end{array}$ & \\
\hline $\begin{array}{l}\text { Multiple } \\
\text { pregnancy }\end{array}$ & & & $<.001$ & & $<0.001$ & & & $<.001$ & & $<.001$ \\
\hline Singleton & $\begin{array}{l}4,617 \\
(49.3 \%)\end{array}$ & reference & & reference & & $\begin{array}{l}5,668 \\
(57.4 \%)\end{array}$ & reference & & reference & \\
\hline Multiple & $\begin{array}{l}375 \\
(83.0 \%)\end{array}$ & $\begin{array}{l}5.02(3.91- \\
6.43)\end{array}$ & & $\begin{array}{l}5.09 \\
6.53)\end{array}$ & & $\begin{array}{l}348 \\
(99.6 \%)\end{array}$ & $\begin{array}{l}5.75(4.20- \\
7.86)\end{array}$ & & $\begin{array}{l}5.68(4.15- \\
7.77)\end{array}$ & \\
\hline Ante-natal care & & & 0.03 & & 0.02 & & & 0.47 & & \\
\hline Yes & $\begin{array}{l}4,876 \\
(50.7 \%)\end{array}$ & reference & & reference & & $\begin{array}{l}5,867 \\
(58.5 \%)\end{array}$ & reference & & reference & \\
\hline No & $\begin{array}{l}116 \\
(58.8 \%)\end{array}$ & $\begin{array}{l}1.36(1.02- \\
1.81)\end{array}$ & & $\begin{array}{l}1.40(1.05- \\
1.86)\end{array}$ & & $\begin{array}{l}149 \\
(60.8 \%)\end{array}$ & $\begin{array}{l}1.10(0.85- \\
1.43)\end{array}$ & & * & \\
\hline
\end{tabular}

Table 4 Univariate and multivariate regression analysis LBW

\section{Discussion}

Our analysis has shown that (pre)-eclampsia rates in the cohort admitted to CRUO between 2013 and 2018 were high. This was not unexpected considering the nature of the hospital was targeted at complicated pregnancies and deliveries. However, it is more than double the rates found in a hospital in rural Haiti [14] and confirms that (pre-) eclampsia rates in Haiti are amongst the highest in the Caribbean [4, 14]. We have also shown that women with (pre-) eclampsia had the highest risk of experienced maternal death, stillbirth and LBW.

The estimated maternal mortality at CRUO over the study period was with 648 women per 100.000 giving birth higher than national mortality rates (529 per 100.000 giving birth) [24]. However, this difference is understandable considering the patient target group for CRUO and strict admission criteria on high-risk pregnancies. No reliable maternal mortality data in obstetric hospitals outside Port-au-Prince is available, but it is likely that these rates are even higher in hospitals in rural areas. This study found that the main risk factor for maternal death was having a C-section, which is in line with global findings $[12,25]$.

We showed that women over the age of 35 were more likely of having a stillbirth regardless of their pregnancy category. This was also shown in a systematic review on maternal age and the risk of stillbirth [18]. Multiple studies demonstrated that C-sections could reduce the number of stillbirths [16, 26 ] and the results in our study echoed this finding. For women who experienced a complicated pregnancy and/or delivery or (pre-) eclampsia, a complicated non-instrumental delivery was associated with the highest risk on stillbirth. However, a substantial proportion of these stillbirths delivered by an obstetric manipulation are considered preventable as early detection of complications and access to quality and timely obstetric care and ANC services can facilitate timely action [27].

The proportion of neonates with LBW in CRUO was with 44.1\% significantly higher than the overall LBW proportion in Haiti (23\%) [4]. We identified that women with (pre-) eclampsia were less likely of having a neonate with LBW if they were under the age of 20, while women with a complicated pregnancy and/or delivery were more at risk of having a neonate with LBW if they were under the age of 20 . Contradictory findings are shown in the literature when assessing the effect of maternal age of having a neonate with LBW. In Finland, advanced maternal age was associated with increased risk of LBW [28], but multiple studies in low- and middle income countries showed that younger maternal age is associated with increased risk of LBW [19, 20]. These associations are most likely confounded by other maternal characteristics, such as maternal diagnosis.

In the total population of this study, $93.4 \%$ of the women indicated they received at least one ANC visit. This proportion was significantly lower in women that experienced a complicated pregnancy and/or delivery (89.0\%), indicating that some potentially complicated pregnancies and deliveries could be 
prevented if women attended ANC services. Not attending ANC services was a risk factor for a stillbirth or having a neonate with LBW when the mother experienced a complicated pregnancy and/or delivery. Women with (pre-) eclampsia did not have a higher risk of having a LBW neonate when they did not attend ANC services. The attendance of ANC services is associated with a decrease in adverse neonatal outcomes including LBW and stillbirth in women with a complicated pregnancy and/or delivery.

Another important finding of this study is the association between delivery procedure and the three negative outcomes. In our population, $40.7 \%$ of the women had a C-section, which were mainly women with a complicated pregnancy and/or delivery (54.5\%). The risk of stillbirth in neonates of women with a complicated pregnancy and/or delivery or (pre-) eclampsia reduced considerably when having a C-section, but it also shows that the risk of maternal death is higher in women with a complicated pregnancy or (pre-) eclampsia when they had a C-section. However, considering the grave clinical condition in which some women arrive at obstetric emergency hospitals, it is probable that they would have died without the $\mathrm{C}$-section being performed. Thus, maternal mortality rates might have been even higher in CRUO if these $\mathrm{C}$-sections would not have been conducted.

Since this is a retrospective study using routinely collected data, we faced numerous challenges in the study. Much of the routinely collected clinical data was not detailed enough to be able to categorize maternal admissions more precisely. Therefore, we were unable to distinguish whether negative outcomes were a (likely) result of a complicated pregnancy or a complicated delivery, or both. This lack of specificity in our dataset therefore also limits the ability to use the results of this study to guide the quality of care in obstetric facilities within Haiti in a more targeted manner. It should also be noted that our results are not generalisable across Haiti, because CRUO targeted mainly high risk pregnancies and complicated deliveries in metropolitan Portau-Prince.

\section{Conclusions}

Our study indicates that maternal mortality remains high in Haiti and that women who do not attend ANC have an increased risk of having a LBW neonate or a stillbirth. Attendance of ANC services is associated with a decrease in adverse neonatal outcomes including LBW and stillbirth and is extremely important to prevent and treat pregnancy complications as early as possible, especially in a context with high blood pressure and high rates of (pre-) eclampsia, like in Haiti. Pre-eclampsia can be detected and appropriately managed before the onset of eclampsia and although the only cure for pre-eclampsia is delivery, ANC could be an opportunity for the provision of aspirin, magnesium and calcium supplementation [16, 29]. We recommend that access to maternal and neonatal healthcare facilities in Port-au-Prince is improved.

\section{Abbreviations}

95\% Cl: 95\% confidence interval; ANC: Antenatal Care; CRUO: Centre de Références des Urgences Obstétricales; C-section: Caesarian section; Cl: Confidence Interval; ERB: Ethical Review Board; LBW: Low Birthweight; OR: Odds Ratio.

\section{Declarations}

\section{Ethics approval and consent to participate}

The study was approved by the National Bioethical Committee of the Ministry of Public Health and Population of Haiti. The study was exempted from approval of the MSF Ethical Review Board (ERB) as it pertained to routinely collected data.

Consent for publication

Not applicable.

\section{Availability of data and materials}

MSF has a managed access system for data sharing. Data are available on request in accordance with MSF's data sharing policy. Requests for access to data should be made to data.sharing@msf.org. For more information please see: 1. MSF's Data Sharing Policy: http://fieldresearch.msf.org/msf/handle/10144/306501 2. MSF's Data Sharing Policy PLOS Medicine article: http://journals.plos.org/plosmedicine/article?id=10.1371/journal.pmed.1001562

\section{Competing interests}

The authors declare that they have no competing interests.

Funding

This study received no specific grant from any funding agency in the public, commercial or not-for-profit sectors. MSF staff carried out the study as part of their routine roles. The corresponding and final authors had full access to all the data and had final responsibility for the decision to submit for publication.

\section{Authors' contributions}


$\mathrm{PF}, \mathrm{ML}$ and $\mathrm{AL}$ were responsible for the study concept and design. Data collection was done by MSF staff on a routine basis and was overseen by $\mathrm{CB}$, RSD and FD. Data cleaning and descriptive analysis was conducted by JS, EB and AL. JS, AL and CA performed the statistical analysis. JS drafted the manuscript and all authors read and approved the final manuscript.

\section{Acknowledgements}

We thank Médecins Sans Frontières, the 'Ministère de la Santé Publique et de la Population' (MSPP) and the National Bioethics Committee for their support of this research. We also thank the CRUO hospital staff involved in data collection for their dedication and hard work.

\section{References}

1. Saleem S, McClure EM, Goudar SS et al. A prospective study of maternal, fetal and neonatal deaths in low-and middle-income countries. Bulletin of the World Health Organization. 2014; 92:605-612. doi: http://dx.doi.org/10.2471/BLT.13.127464

2. Bhutta ZA, Black RE. Global maternal, newborn, and child health-so near and yet so far. N Engl J Med. 2013; 369(23):2226-35. http://dx.doi.org/10.1056/NEJMra1111853 pmid: 24304052

3. Ramachandran V, Walz J. Haiti: Where Has All the Money Gone? Journal of Haitian Studies. 2015; 21 (1): 26-65

4. Jacobs LD, Judd TM, Bhutta ZA. Addressing the child and maternal mortality crisis in Haiti through a central referral hospital providing countrywide care. Perm J. 2016; 20(2):59-70

5. United Nations Inter-agency Group for Child Mortality Estimation (UN IGME). 2018. http://data.unicef.org

6. United Nations Children's Fund. Health Equity Report 2016: Analysis of reproductive, maternal, newborn, child and adolescent health inequities in Latin America and the Caribbean to inform policymaking. Summary Report. 2016.

7. World Bank, Global Poverty Working Group. National official poverty line developed in 2012. https://databank.worldbank.org/data/reports.aspx? source=2\&country $=$ HTI\&series $=$ \&period

8. Amibor P. What will it take to maintain the maternal and child health gains made in Haiti prior to the 2010 earthquake?: An analysis of past progress, trends, and the prospects for the realization of the United Nations Millennium Development Goals 4 and 5 . Maternal and Child Health Journal. 2013; 17(8), 1339-1345. doi:10.1007/s10995-012-1170-0

9. Hutchinson K, Bryant M, Bachman De Silva M et al. Delayed access to emergency obstetrical care among preeclamptic and nonpreeclamptic women in Port-Au-Prince, Haiti. BMC Pregnancy and Childbirth. 2018; 18:337 https://doi.org/10.1186/s12884-018-1961-4

10. Streeter BM. Maternal Mortality in Haiti. Senior Honors Theses. 2017; 180. http://digitalcommons.brockport.edu/honors/180

11. Santana DS et al. Twin Pregnancy and Severe Maternal Outcomes. Gynecol. Apr 2016; 127(4): 631-641.

12. Briand V et al. Maternal and Perinatal Outcomes by Mode of Delivery in Senegal and Mali: A Cross-Sectional Epidemiological Survey. PLoS One. 2012; 7(10): 6-13.

13. Campbell OM, Graham WJ. Lancet maternal survival series steering committee: strategies for reducing maternal mortality: getting on with what works. Lancet. 2006; 368(9543): 1284-99.

14. Raghuraman N, March MI, Hacker MR et al. Adverse maternal and fetal outcomes and deaths related to preeclampsia and eclampsia in Haiti. Pregnancy Hypertension. 2014; 4(4): 279-286

15. Mirkovic KR, Lathrop E, Hulland EN et al. Quality and uptake of antenatal and postnatal care in Haiti. BMC Pregnancy and Childbirth. 2017. DOI $10.1186 / \mathrm{s} 12884-016-1202-7$

16. Feresu SA, Harlow SD, Welch $\mathrm{K}$ et al. Incidence of stillbirth and perinatal mortality and their associated factors among women delivering at Harare Maternity Hospital, Zimbabwe: a cross-sectional retrospective analysis. BMC Pregnancy and Childbirth. 2005, 5:9 doi:10.1186/1471-2393-5-9

17. Kahn B, Lumey LH, Zybert PA et al. Prospective risk of fetal death in singleton, twin and triplet gestations : implications for practice. Obstet Gynecol. 2003; 102(4):685-92.

18. Huang L, Sauve R, Birkett N et al. Maternal age and risk of stillbirth: a systematic review. 2008; 178 (2) 165-172. https://doi.org/10.1503/cmaj.070150

19. Ganchimeg T, Ota E, Morisaki N et al. Pregnancy and childbirth outcomes among adolescent mothers: a World Health Organization multicountry study. BJOG. 2014; 121, 40-48. https://doi.org/10.1111/1471-0528.12630

20. Chen XK, Wen SW, Fleming $\mathrm{N}$ et al. Teenage pregnancy and adverse birth outcomes: a large population based retrospective cohort study. International Journal of Epidemiology. 2007; 36 (2): 368-373, https://doi.org/10.1093/ije/dyl284

21. Shinwell ES. Neonatal and long-term outcomes of very low birth weight infants from single and multiple pregnancies. Semin Neonatol. 2002; 7:203209.

22. Muhuri PK, MacDorman MF, Menacker F. Method of delivery and neonatal mortality among very low-birthweight infants in the United Stated. Maternal and Child Health Journal. 2006; 10(1) DOI: 10.1007/s10995-005-0029-z

23. WHO, “Family Planning Maternal \& Child Health and Reproductive Health," 2001.

24. Institut Haïtien de l'Enfance (IHE) et ICF. Enquête Mortalité, Morbidité et Utilisation des Services (EMMUS-VI 2016-2017). 
25. Souza JP et al., "C-section without medical indications is associated with an increased risk of adverse short-term maternal outcomes: the 20042008 WHO Global Survey on Maternal and Perinatal Health," BMC Med. 2010; 8 (71): 1-10

26. McClure EM, Goldenberg RL. Stillbirth in developing countries: a review of causes, risk factors and prevention strategies. J Matern Fetal Neonatal Med. 2009; 22(3): 183-190. doi:10.1080/14767050802559129.

27. Buinoiu NF, Stoica SI, Mat C et al. Mode of Delivery in Stillbirth. Maedica (Buchar). 2017; 12(2):101-105.

28. Goisis A, Remes H, Barclay K et al. Advanced Maternal Age and the Risk of Low Birth Weight and Preterm Delivery: a Within-Family Analysis Using Finnish Population Registers. American Journal of Epidemiology. 2017; 186 (11): 1219-1226, https://doi.org/10.1093/aje/kwx177

29. Duley L, Gülmezoglu AM, Henderson-Smart DJ, Chou D. Magnesium sulphate and other anticonvulsantsfor women with pre-eclampsia. Cochrane Database Syst Rev. 2010; 11:CD000025. https://doi.org/10.1002/14651858.CD000025.pub2 PMID: 21069663 